Seguridad Operacional y Logística Aeronáutica

Segurança Operacional e Logística Aeronáutica

Operational Safety and Aviation Logistics

\title{
Vigilancia Dependiente Automática (ADS-B) en Colombia ${ }^{1}$
}

\author{
Vigilância Dependente Automática (ADS-B) na Colômbia² \\ Automatic Dependent Survelllance (ADS-b) in Colombia 3 \\ Leonardo Gómez ${ }^{4}$ \\ Centro de Estudios de Ciencias Aeronáutica de la Aeronáutica Civil de Colombia
}

CIENCIA Y PODER AÉREO

ISSN 1909-7050 / E- ISSN 2389-2468 / Volumen 10 / Enero-Diciembre de 2015/ Colombia/ Pp. 1-260

Recibido: 30/09/2015

Aprobado evaluador interno: 12/10/2015

Aprobado evaluador externo: 30/11/2015

DOl: http://dx.doi.org/10.18667/cienciaypoderaereo.215 
CIENCIA Y PODER AÉREO | Revista Científica de la Escuela de Postgrados de la Fuerza Aérea Colombiana | Vol. 10 | Enero - Diciembre de 2015

Para citar este artículo:

Gómez, L. (2015). Vigilancia Dependiente Automática (ADS-B) en Colombia. Ciencia y Poder Aéreo, 10 (1), 21-32. Doi: http://dx.doi.org/10.18667/cienciaypoderaereo.215

\footnotetext{
'Artículo científico original derivado del trabajo investigativo desarrollado y financiado por el Centro de Estudios de Ciencias Aeronáutica de la Aeronáutica Civil de Colombia (CEA).
}

${ }^{2}$ Artigo científico original derivado do trabalho de pesquisa realizado e financiado pelo Centro para o Estudo das Ciências Aeronáuticas da aeronáutica Civil na Colômbia (CEA).

${ }^{3}$ Original scientific article derived from research work carried out and funded by the Center for Aeronautical Sciences Studies of the Colombian Civil (CEA).

${ }^{4}$ Ingeniero Electrónico egresado de la Universidad Distrital, Especialista en Gerencia de Proyectos en Ingeniería, Magíster en Ingeniería de Telecomunicaciones de la Universidad Nacional de Colombia con mención meritoria de tesis. Instructor del Grupo ATSEP (Air Traffic Safety Electronic Personnel) en el Centro de Estudios de Ciencias Aeronáuticas C.E.A de Aeronáutica Civil de Colombia. Docente del programa de Ingeniería Aeronáutica de la Universidad de San Buenaventura sede Bogotá. Correo electrónico: leonardo_54@hotmail.com
Resumen: en este artículo se presentan las actuales tecnologías usadas para la prestación de los servicios de navegación y vigilancia aeronáutica en Colombia. También, se realiza una breve descripción del concepto Communication, Navigation, Surveillance/Air Traffic Management (CNS/ATM), haciendo énfasis en la tecnología Automatic Dependent Surveillance-Broadcast (ADS-B), con lo que se busca analizar las ventajas y desventajas que trae consigo su implementación, teniendo en cuenta las condiciones de tránsito aéreo y geografía colombiana. ADS-B es la tecnología mediante la cual las aeronaves, vehículos de aeródromo, y otros objetos pueden automáticamente transmitir y/o recibir datos de identificación, posición en cuatro dimensiones, y datos de los sistemas de posicionamiento y navegación. El objetivo principal de este artículo es presentar el estado del servicio ADS-B en Colombia, y mostrar cuáles son las tecnologías que están siendo usadas para su implementación.

Palabras clave: Área de Control Terminal TMA; ADS-B; CNS/ATM; enlaces de datos; servicios a la navegación aérea; vigilancia aeronáutica.

Resumo: neste artigo são apresentadas as atuais tecnologias utilizadas para a prestação de serviços de navegação e de vigilância aeronáutica na Colômbia. Também é apresentada uma breve descrição do conceito CNS/ATM, enfatizando na tecnologia de Vigilância Dependente Automática - Broadcast (ADS-B). Esta descrição é para analisar as vantagens e desvantagens que vêm com a implementação de novas tecnologias para a prestação de serviços de navegação aérea, em especial, ADS-B, tendo em conta as condições de tráfego aéreo e a geografia colombiana. O principal objetivo deste artigo é apresentar o status do serviço ADS-B, na Colômbia, e as tecnologias que estão sendo usadas para sua implementação.

Palavras-chave: ADS-B; CNS / ATM; links de dados; serviços de navegação aérea; vigilância aérea.

Abstract: This paper presents an overview of current technologies used to provide air navigation and surveillance services in Colombia. It also presents the CNS/ATM (Communication, Navigation, Surveillance/Air Traffic Management) concept, with emphasis on the Automatic Dependent Surveillance - Broadcast (ADS-B). This is done in order to analyze the advantages and disadvantages that entail the implementation of new technologies for air traffic services, in particular ADS-B, taking into account the Colombian geography and air traffic special conditions. Its main goal, is to present the state of implementation of ADS-B service in Colombia, and to show technologies which are being used to this purpose, as antennas, receivers and data link protocols.

Key Words: Air Navigation Services; Automatic Dependent Surveillance; CNS/ATM; Data Links; Surveillance; Terminal area TMA. 


\section{Introducción}

El objetivo principal de este artículo es presentar el estado del servicio ADS-B en Colombia, y mostrar cuáles son las tecnologías que están siendo usadas para su implementación. Se comienza estableciendo las condiciones de tránsito aéreo y espacio aéreo colombiano, para luego describir las tecnologías que están siendo usadas actualmente para la prestación de los servicios de navegación y vigilancia aeronáutica. También, se describen en general diferentes tecnologías que se planean implementar por parte de la autoridad de aviación civil en Colombia, haciendo énfasis en ADS-B para la prestación del servicio de vigilancia.

La actual infraestructura utilizada para la prestación de los servicios a la navegación aérea en Colombia, está basada en radio ayudas convencionales como: VOR, DME, ILS, $N D B$, y la infraestructura para el servicio de vigilancia está basada en Radares Primarios (PSR) y Secundarios (SSR) (Matamoros, 1999). Debido al incremento de las operaciones aéreas en Colombia en un futuro cercano, se espera que estas tecnologías sean insuficientes para las necesidades generadas por el aumento en el número de aeronaves. Este incremento se evidencia en las tablas 5, 6 y 7 del volumen 1 del Plan Nacional de Navegación Aérea para Colombia (PNA) (Aeronáutica Civil de Colombia, 2014), y puede verse en la Figura1.

Debido a este incremento en las operaciones aéreas, ha sido establecido por la Aeronáutica Civil que para la prestación de los servicios de navegación y vigilancia aeronáutica, es requerida una transición de tecnologías a sistemas basados en satélites, sistemas de aumentación y enlaces de datos, entre otra serie de aplicaciones documentadas en el PNA (OACI GREPECAS, 2011). Este documento establece dicha transición a corto, mediano y largo plazo hacia tecnologías cuyo fundamento son los enlaces de datos, la automatización y el uso de satélites para los servicios de comunicaciones, navegación, vigilancia y meteorología. Con estas tecnologías se pueden prestar servicios como Vigilancia Dependiente Automática (ADS), Comunicaciones vía Enlaces de datos entre Controlador y Piloto (Controller Pilot data Link Communication (CPDLC)), Servicios de información de vuelo vía enlaces de datos (Data Link flight information Services (DFIS)), entre otros (Gómez y Ortíz, 2012; Farroha, 2002).

Actualmente, en Colombia ya se ha iniciado la implementación de varias estaciones de referencia en tierra con capacidad para la prestación del servicio ADS-B. Su propósito es proveer servicios de navegación y vigilancia para las aeronaves que sobrevuelen el espacio aéreo colombiano. Algunas estaciones de referencia en tierra ya se han instalado, y otras serán instaladas a corto plazo. Se espera que en

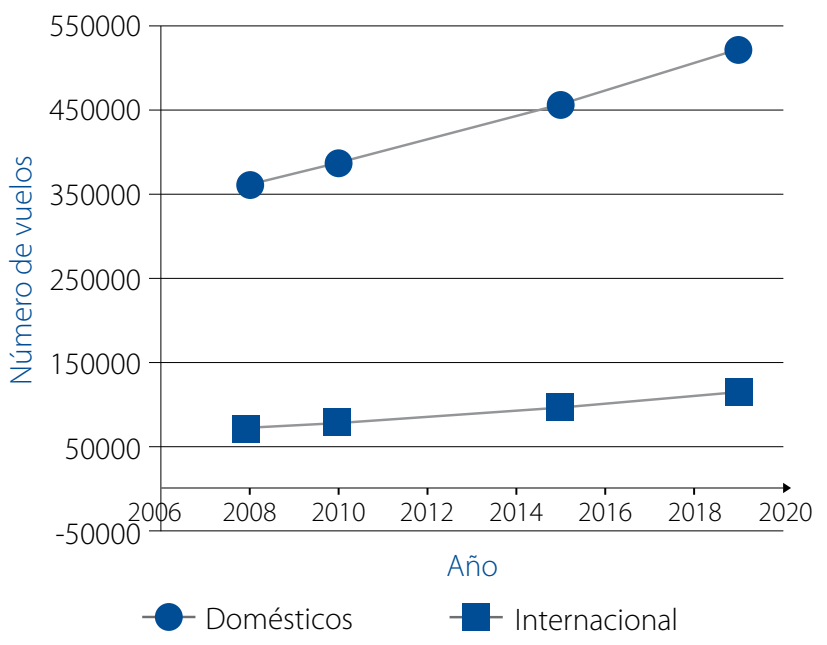

Figura 1. Incremento del tránsito aéreo en Colombia 2008-2019. Fuente: Gómez \& Ortíz. (2013). Modeling and simulation of VDL mode 2 subnet for CPDLC in El Dorado Airport. 32st Digital Avionics Systems Conference, DASC.

el futuro cercano, estas complementen a los sistemas de Radar usados actualmente, para proveer el servicio de vigilancia en lugares donde la señal radar no tiene cobertura en la actualidad, sin necesidad de recurrir a la implementación de costosos sistemas PSR y SSR.

La vigilancia con sistemas Radar, funciona enviando ondas electromagnéticas desde complejas estaciones en tierra hasta los blancos móviles, y luego interpretando las ondas reflejadas en ellos (Mahafza, 1998). En lugar de esto, ADS-B usa el Sistema Global de Navegacion por Satélite (GNSS) para localizar la aeronave, y enlaces de datos en Broadcast para informar dicha posición a los sistemas de vigilancia en tierra, como sus principales componentes de operación (Airservices Australia, 2012). Contrario a los actuales sistemas de vigilancia, la precisión de ADS-B no se degrada con el alcance, condiciones atmosféricas o la altitud de los blancos móviles, y los intervalos de actualización de la información de posición no depende del movimiento rotatorio de la antena de la estación en tierra. Las aeronaves con capacidad ADS-B usan receptores GPS, GLONASS o Galileo para determinar su posición con la constelación GNSS, y combina esta información con la velocidad, rumbo, altitud, identificación, entre otros. Esta información es entonces enviada simultáneamente a otras aeronaves con capacidad ADS-B, estaciones en tierra o transceptores satelitales que se encuentren dentro del alcance del transmisor a bordo, los cuales entregan la información a los centros de control de tránsito aéreo en tiempo real (ADS-B Technologies, 2015; OACl, 1999; Richards y Miller , 2014). 


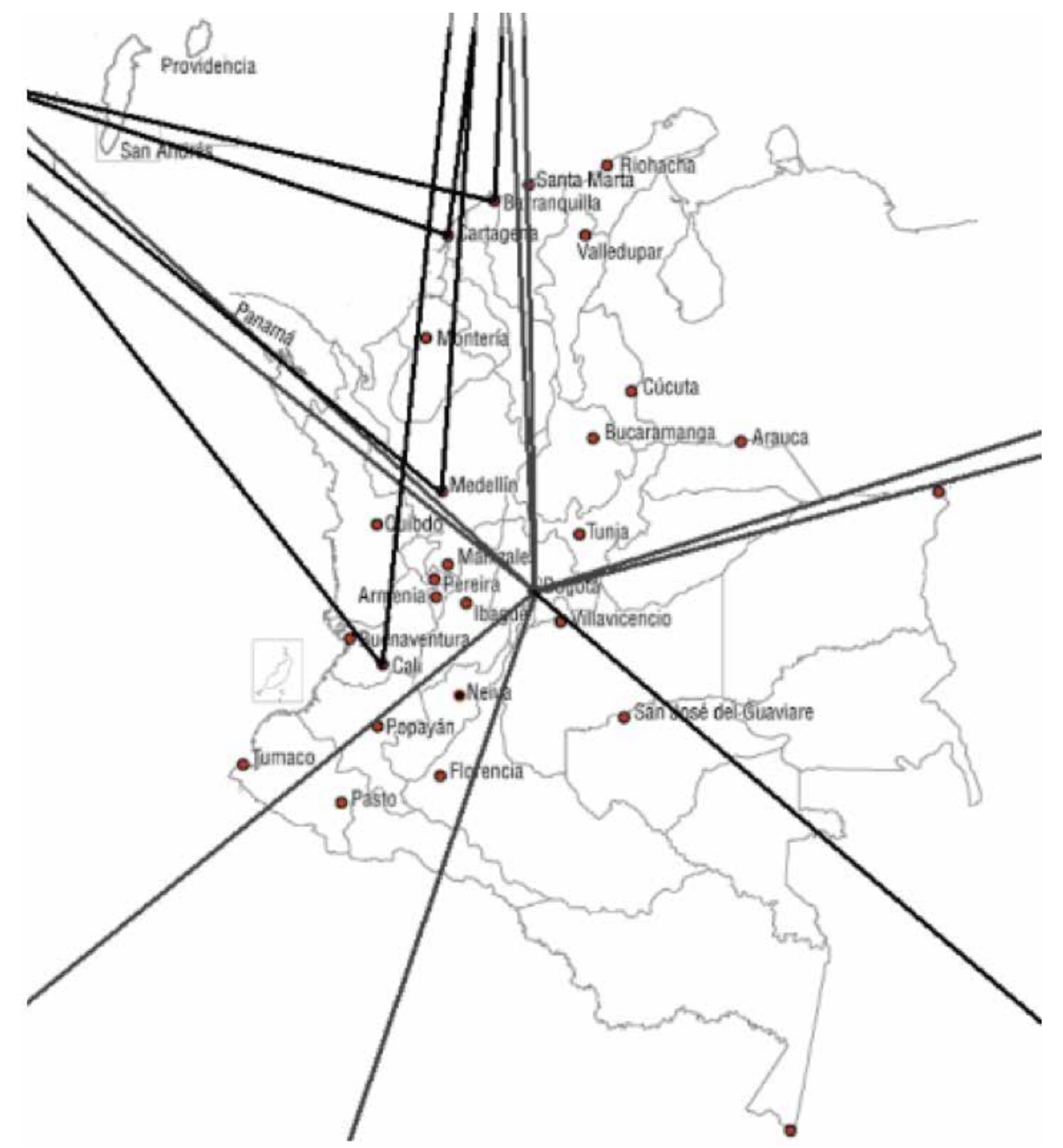

Figura 2. Rutas internacionales sobre Colombia. Fuente: Aeronáutica Civil de Colombia (2011). Plan de navegacion aérea para Colombia. Servicios e Instalaciones (Vol. 2). Bogotá.

\section{Condiciones de tránsito aéreo en Colombia}

Colombia tiene una ubicación geográfica excepcional para las operaciones de tránsito aéreo entre Norte, Centro y Sur América, además de las operaciones desde Sur América hacia Europa y viceversa. Esta posición le permite manejar cerca del $75 \%$ del tráfico en estas rutas y le da una ventaja a Colombia sobre sus países vecinos. En la figura 2, pueden verse las rutas internacionales sobre el territorio colombiano (Aeronáutica Civil, 2013).

Los principales aeropuertos de Colombia se localizan sobre la cordillera de los Andes, la cual cruza el territorio colombiano de norte a sur. Esta posición natural crea difíciles condiciones para la localización de radio ayudas a la navegación aérea, estaciones terrenas de comunicaciones, y antenas de vigilancia radar con el objetivo de obtener la mayor cobertura posible dentro del espacio aéreo colombiano (Aeronautica Civil, 2015).

Debido a esto, las rutas aéreas y el diseño de procedimientos de navegación, están altamente influenciados por la forma de las cordilleras, la ubicación de los aeropuertos dentro de ellas, y la posición de las estaciones de comunicaciones, navegación y vigilancia en tierra. La Figura 3 muestra las rutas domésticas dentro del espacio aéreo colombiano.

\section{Espacio aéreo colombiano}

El espacio aéreo superior cubre la totalidad del país incluyendo las áreas marítimas sobre $20.000 \mathrm{ft}$. Los servicios de control de tránsito aéreo son prestados en la totalidad de 


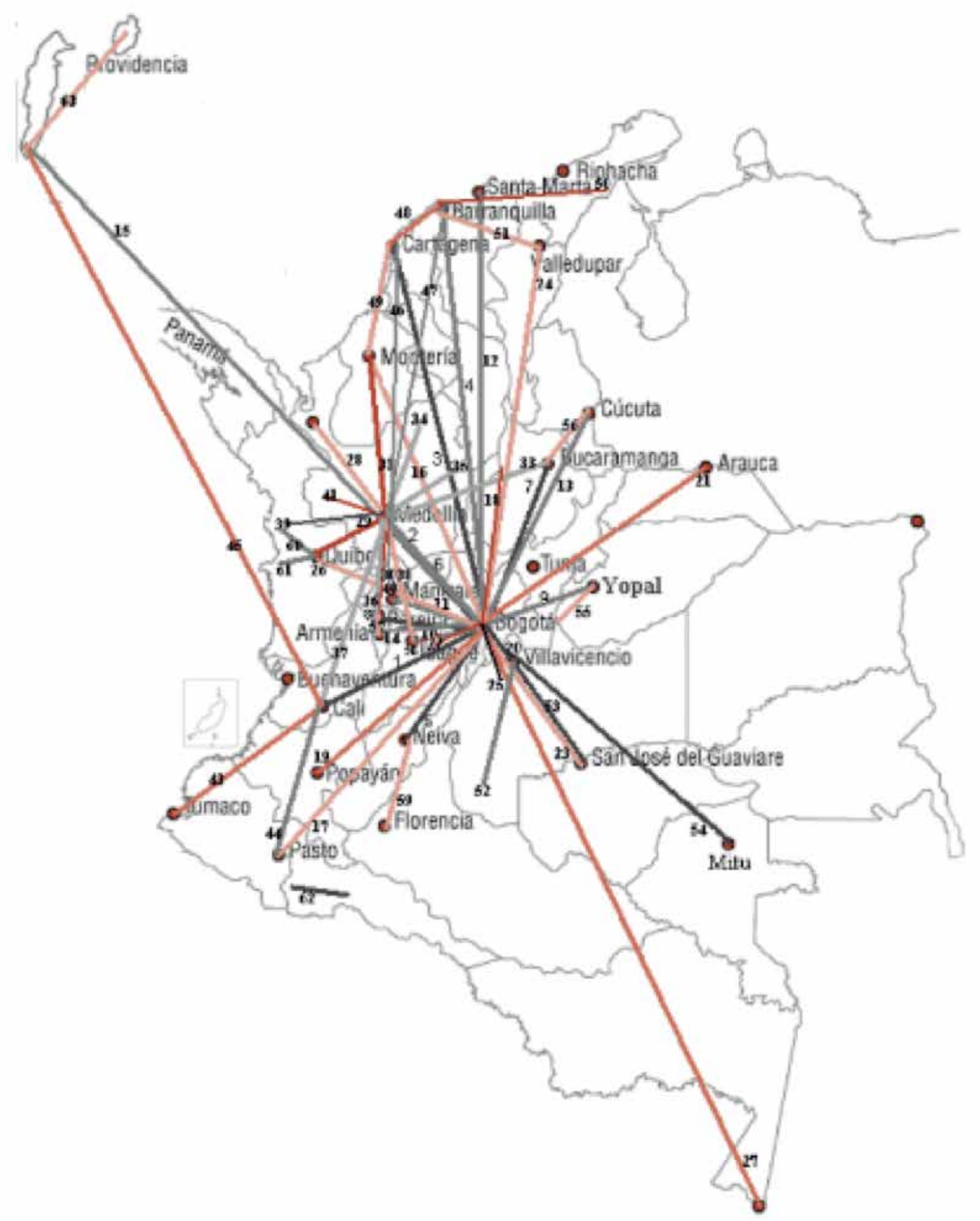

Figura 3. Rutas domésticas sobre Colombia.

Fuente: Aeronáutica Civil de Colombia (2011). Aeronáutica Civil de Colombia. (2011). Plan de navegacion aérea para Colombia. Servicios e Instalaciones (Vol. 2). Bogotá.

este espacio, el cual está dividido en secciones llamadas Flight Information Regions (FIR). Colombia está dividido en dos regiones: FIR Bogotá (BOG FIR) y FIR Barranquilla (BAQ FIR) como se muestra en la Figura 4 (Gómez y Ortíz , 2013; Aeronautica Civil, 2015).

\section{FIR Bogotá}

El centro de control de esta FIR está localizado en la ciudad de Bogotá. Su área total se divide en cuatro sub sectores, de los cuales cada uno de ellos tiene una frecuencia independiente de comunicaciones como se muestra en la Figura 4. El sistema que permite las comunicaciones en- tre los controladores de tránsito aéreo y las aeronaves que vuelan dentro de cada subsector, hace parte de la llamada red en VHF de alcance extendido VHF-ER. Alrededor de seis o siete estaciones remotas son usadas por sector para lograr una cobertura total de comunicaciones dentro de la región FIR (Gómez y Ortíz , 2013).

\section{FIR Barranquilla}

El centro de control de esta región FIR está localizado en la ciudad de Barranquilla. Su área total está dividida en dos sectores, y como en la FIR Bogotá, cada sector tiene una frecuencia independiente para la prestación de los 


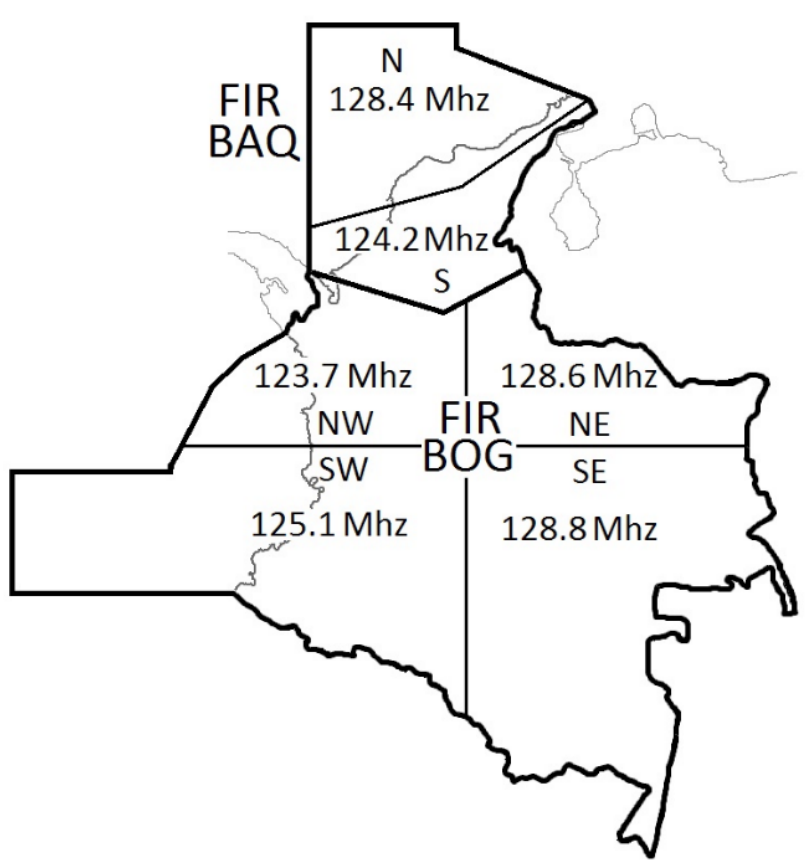

Figura 4. Espacio aéreo superior colombiano.

Fuente: Gómez \& Ortíz. (2014). Results of a VDL 2 subnet simulation in the approach area to El Dorado Airport. 33st Digital Avionics Systems Conference, DASC.

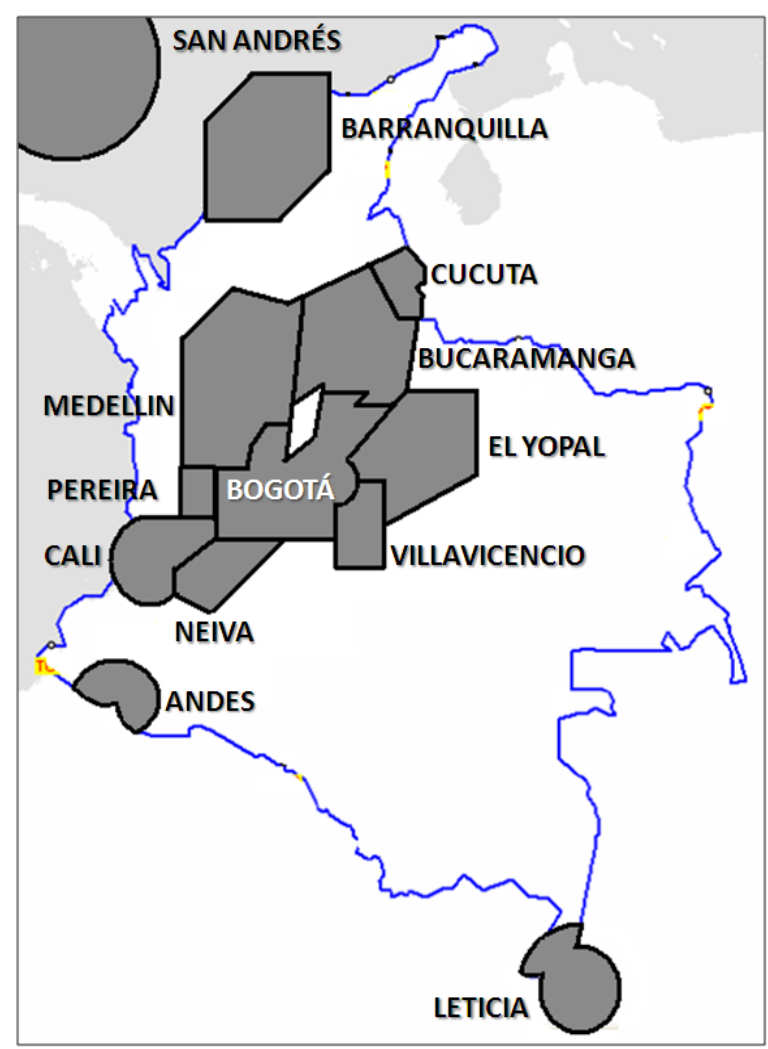

Figura 5. Espacio aéreo inferior colombiano.

Fuente: Aeronáutica Civil. (2014). Plan de Navegación Aérea para Colombia (Vol. 1). Bogotá. servicios de navegación aérea como se ve en la Figura 4. También, se usan entre seis o siete estaciones en tierra por sector para lograr una cobertura total de comunicaciones dentro de cada espacio aéreo.

\section{Espacio aéreo inferior}

En el espacio aéreo inferior de Colombia se tienen 13 áreas de control terminal (TMA). Estas son áreas controladas, diseñadas alrededor de los principales aeropuertos. Generalmente un área TMA tiene una forma circular alrededor de las coordenadas geográficas de un aeropuerto, pero debido a la geografía y la proximidad entre los principales aeropuertos colombianos, estas trece TMAs toman variadas formas (OACl, 2013). Algunas de ellas están divididas en sectores más pequeños, cada uno de los cuales tiene asignada un canal de comunicaciones en VHF para las comunicaciones aire-tierra. En la Figura 5 se pueden ver las TMAs dentro del territorio colombiano.

\section{Tecnologías usadas actualmente para la prestación de los servicios a la navegación aérea en Colombia}

\section{Vigilancia RADAR}

Durante los últimos años, los sistemas de Radar Primario (PSR) y de Radar Secundario (SSR) han tenido un importante desarrollo, siendo los principales sistemas usados para la prestación del servicio de vigilancia en Colombia. La cobertura de la señal de los radares está restringida a las áreas continentales y costeras. Un alto porcentaje de las regiones oceánicas se encuentran fuera del alcance de los sistemas de Radar. La ubicación de las estaciones Radar en tierra en el territorio colombiano pueden verse en la Figura 6 (Gómez y Ortíz, 2012; Hamish, 2001).

Los servicios a la navegación aérea deben ser soportados por sistemas que entreguen los más altos estándares de calidad, disponibilidad, integridad y confiabilidad en todas las estaciones aeronáuticas y en todos los aeropuertos donde se realicen operaciones de tránsito aéreo. Teniendo esto en cuenta, la Aeronáutica Civil de Colombia implementó la red nacional de vigilancia aeronáutica en la década de los 90's, la cual incluye sensores Radar, sistemas de procesamiento, unidades de visualización de datos radar, y sistemas de administración en las estaciones de control ATC, para su uso por parte del servicio de control de tránsito aéreo $(\mathrm{OACl}, 2007)$. La red nacional de radares se ha estado expandiendo desde los 90's hasta ahora, de tal forma que actualmente se cuenta con 20 estaciones de Radar (11 Radares de vigilancia secundario (SSR) y 9 Radares de vigilancia Primarios (PSR)) (Aeronáutica Civil, 2011). 


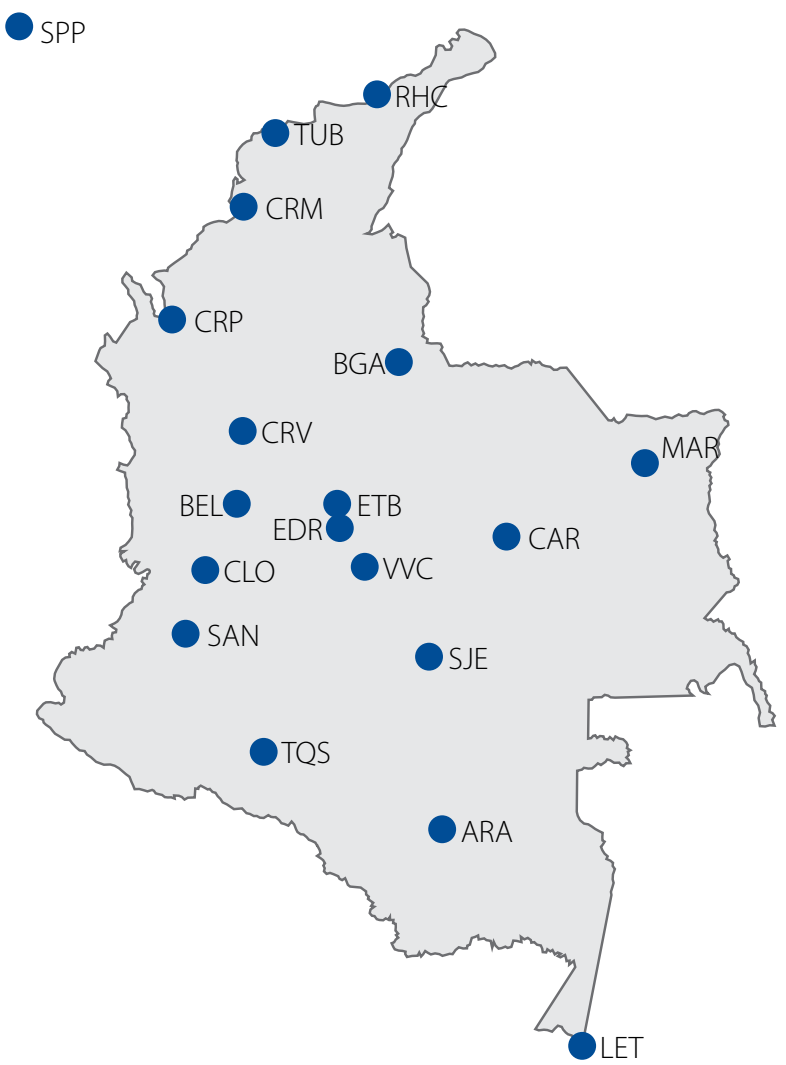

Figura 6. Posición de las estaciones Radar.

Fuente: elaboración del autor.

\section{Radio ayudas para la navegación aérea}

La infraestructura para la aviación en el campo de la navegación en Colombia, está basada en radio ayudas en tierra (OACl, 2007). Estas radio ayudas, proveen suficiente cobertura permitiendo la realización de vuelos seguros sobre las aerovías diseñadas para tal fin. Sin embargo, las radio ayudas en Colombia presentan una baja densidad en su distribución debido a las especiales condiciones de la geografía colombiana a lo largo de sus diferentes regiones, en especial en regiones como la Amazónica y la Oceánica. En estas dos últimas, una cobertura apropiada es difícil de lograr. Actualmente, la infraestructura de radio ayudas está conformada por 50 sistemas VOR, 52 DME (50 asociados a los VOR + 2 independientes), 11 sistemas ILS y 18 sistemas NDB (Aeronáutica Civil, 2011).

Con esta infraestructura se presta el servicio de navegación en las diferentes fases de cada vuelo. Los equipos Non Directional Beacon (NDB) son sistemas que proveen información de posición horizontal de no precisión, indicando el radial de la dirección de las aeronaves en fase de ruta y aproximación. Los VHF Omnidirecional Range (VOR), son sistemas que proveen también información de posición horizontal de no precisión indicando los radiales en todas las direcciones para ser seguidos por aeronaves en las fases de ruta y aproximación. El Distance Measuring
Equipment (DME) es un sistema que provee la distancia oblicua en millas náuticas (NM) desde la aeronave hasta la estación en tierra. El Instrument Landing System (ILS) provee a la aeronave información exacta de posición tanto horizontal como vertical en las fases de aproximación y aterrizaje. Esto lo hace indicándole al piloto si el avión está alineado con el eje principal de la pista y el ángulo de descenso hasta la Touch Down Zone (TDZ). Dentro de los sistemas disponibles en Colombia para la navegación aérea, se encuentra el Sistema Global de Navegación por Satélite (GNSS). Aquellas aeronaves con la aviónica básica de navegación GPS, pueden volar procedimientos de no precisión en fases de ruta y aproximación.

\section{Comunicaciones, Navegación y Vigilancia en CNS/ ATM}

En 1983, el Concejo de la Organización de Aviación Civil Internacional $(\mathrm{OACl})$, determinó que los sistemas y procedimiento que soportaban la aviación civil habían alcanzado sus límites, y estableció el Comité Especial para los Sistemas de Navegación Aérea del Futuro. (FANS - Future Air Navigation Systems). Al concluir su trabajo en 1989, el comité FANS determinó que sería necesario desarrollar nuevos sistemas que superaran las limitaciones de los sistemas convencionales y entonces alcanzar el desarrollo de los sistemas para la gestión del tránsito aéreo (ATM) en todo el mundo. En julio de 1989, el concejo de la OACl estableció el comité especial para el monitoreo y la coordinación del desarrollo y plan de transición para los sistemas de navegación aérea del futuro. (Comité FANS fase 2). En octubre de 1993 este segundo Comité concluyó su trabajo reconociendo que la aplicación de nuevas tecnologías y sus beneficios deberían desarrollarse durante un determinado periodo de tiempo. En septiembre de 1991. Fue presentado en la décima Conferencia de Navegación Aérea el concepto de los futuros sistemas para la navegación aérea, desarrollados por los Comités FANS. Este concepto envuelve una serie de complejos conceptos relacionados con tecnologías dependientes de satélites y que hacen alto uso de enlaces de datos con el objetivo de automatizar la Gestión del Tránsito Aéreo (Gómez y Ortíz, 2012; OACl, 2012; Morales Lopez, 2001).

\section{Beneficios de los sistemas CNS/ATM}

Los sistemas CNS/ATM mejoran el manejo y la transferencia de información, extienden la vigilancia y mejoran la precisión para la navegación. Lo anterior permite una reducción en la separación entre aeronaves lo cual redunda en mayor capacidad del espacio aéreo. Avanzados sistemas CNS/ATM en tierra intercambian información directamente con los Flight Management Systems (FMS) a bordo de la aeronave a través de subredes de enlaces de datos. Esto conlleva un beneficio para los prestadores de servicios ATM y usua- 
CIENCIA Y PODER AÉREO | Revista Científica de la Escuela de Postgrados de la Fuerza Aérea Colombiana | Vol. 10 | Enero - Diciembre de 2015

rios del espacio aéreo, permitiendo la detección de conflictos a través de procesamiento inteligente de la información, y la generación y transmisión automática de mensajes libres de conflictos, así como una comunicación que se adapte rápidamente a los cambios o necesidades del tránsito aéreo. Como resultado de esto, el sistema ATM tendrá la capacidad de acomodar el perfil de vuelo de una aeronave seleccionada y ayudar a los operadores a reducir las demoras en los vuelos y los costos de operación (OACl, 2012).

\section{Comunicaciones}

Dentro del concepto CNS/ATM, un enlace de datos es uno de los elementos clave junto con tecnologías satelitales y automatización. De hecho, estos enlaces permiten la posibilidad de proveer servicios de navegación, vigilancia e intercambios de información con datos, gráficos e imágenes en lugar de solamente mensajes de voz (OACl, 2002).

Las comunicaciones vía enlaces de datos soportan servicios como CPDLC, ADS, DFIS y en general intercambios de información entre las aeronaves y los sistemas ATC en tierra. Estos últimos, mejoran el manejo de la información y transferencia entre operadores, aeronaves, y centros de control. Esta mejora le permite al piloto acceder a los diferentes servicios provistos por la red de telecomunicaciones aeronáuticas (ATN) desde su computador a bordo (OACl, 2002).

\section{Navegación}

La información acerca de la posición de una aeronave es la condición básica para que esta pueda navegar. Desde hace mucho tiempo, los sistemas convencionales basados en estaciones de referencia en tierra han provisto señales de radiofrecuencia mediante las cuales los sistemas de abordo pueden calcular la posición de la aeronave en coordenadas polares (acimut, distancia y altitud) con respecto a dichas estaciones. Las desventajas que tienen estos sistemas convencionales, se evidencian en la infraestructura rígida de las actuales rutas ATS que dependen de su localización. Estas rutas están determinadas por el lugar donde las radio ayudas se encuentren instaladas. En este sentido, el sistema es inflexible en términos de geografía, así como en términos de rutas y capacidad. En el concepto CNS/ ATM, el posicionamiento y la navegación se relacionan con el Sistema (GNSS) y conceptos como Navegación de Área (RNAV - Area Navigation) y Performance de Navegación requerido (PBN - Requirement Navigation Performance), generando flexibilidad a los diseñadores de procedimientos y usuarios del espacio aéreo (Gómez y Ortíz, 2012).

Las constelaciones de satélites usadas para navegación aérea, como NAVSTAR-GPS de los Estados Unidos y Global'naya Navigatsionnaya Sputnikovaya System (GLONASS) administrada por Rusia, entre otras constelaciones, no cumplen con la totalidad de requerimientos específicos para procedimientos de navegación en aviación civil, especialmente en procedimientos de precisión como una aproximación o un aterrizaje. El funcionamiento erróneo de cualquiera de los satélites de la constelación, o interferencias aleatorias causadas por la ionósfera o fenómenos del espacio o del tiempo meteorológico, afectan a los usuarios de un determinado espacio aéreo. Estas afectaciones decrementan los niveles de precisión y por lo tanto, los niveles de seguridad. Es por esto que se hace necesario complementar los servicios provistos por las constelaciones con elementos adicionales que hacen parte del sistema GNSS, llamados Sistemas de Aumentación.

Los Sistemas de Aumentación incrementan la precisión de la posición obtenida por una aeronave o un proveedor de servicios de tránsito aéreo. Satellite Based Augmentation System (SBAS), Ground Based Augmentation System (GBAS) y Aircraft Based Augmentation System (ABAS) son los tres sistemas de aumentación propuestos a la comunidad de aviación a nivel mundial. Estos sistemas mejoran la precisión de la información, corrigiendo el error que se pueda presentar en determinado momento usando dispositivos localizados en tierra, o satélites adicionales (Aeronáutica Civil, 2014; Aeronáutica Civil, 2011).

\section{Vigilancia}

En el concepto CNS/ATM, el servicio de vigilancia hace referencia al intercambio aire-tierra de información sobre posición, entre los computadores de abordo y de tierra. Los controladores de tránsito aéreo obtienen la información sobre la situación del espacio aéreo en una pantalla similar a la de Radar, aun más allá del rango de cobertura de los sistemas de vigilancia convencionales. La vigilancia es optimizada a través de la utilización de tecnologías como ADS. Esta tecnología se trata de un sistema, que haciendo uso de un enlace de datos, entrega la información de la posición de la aeronave obtenida del sistema de posicionamiento global GNSS abordo, automáticamente a la estación en tierra más cercana, la cual la envía hasta el centro de control con alta velocidad y precisión.

\section{Automatic Dependent Surveillance - Broadcast (ADS-B)}

El ADS-B es la tecnología mediante la cual las aeronaves, vehículos de aeródromo, y otros objetos pueden automáticamente transmitir y/o recibir datos de identificación, posición en cuatro dimensiones, y datos de los sistemas de posicionamiento y navegación, a través de enlaces de datos (OACl, 1999). ADS-B mejora significativamente la calidad de la información con respecto a la entregada por los sistemas de Radar basados en estaciones terrenas. De esta manera, está llamada a reemplazar los 
sistemas convencionales. El ADS-B es automático, porque no necesita estímulos externos. Es dependiente (o colaborativo), porque la aeronave debe entregar la información de su posición para que las estaciones en tierra puedan prestar el servicio de vigilancia. Finalmente, la aeronave transmite los datos sin saber quién va a recibir y usar la información, ya que no existe un acuerdo de reciprocidad o interrogación. Debido a esto es que se le denomina Broadcast. Los detalles técnicos y las especificaciones detalladas de esta aplicación se encuentran contenidas en el documento 9705 de la OACl.

De acuerdo con la OACI, ADS-B es una aplicación de vigilancia que permite la transmisión de parámetros, como posición e identificación, a través de un enlace de datos en modo broadcast, para ser usado por cualquier estación de tierra o aire que los requiera. Esta aplicación no está limitada a los tradicionales roles asociados con los sistemas de Radar basados en tierra. ADS-B proporciona oportunidades para nuevas funcionalidades tanto a bordo como en tierra en los sistemas automatizados de ATC. Dependiendo de su implementación, ADS-B puede sincronizar funcionalidades tanto aire-tierra, como aire-aire, además de aplicaciones aeronaves o entre los diferentes vehículos de un aeródromo. Otro de los beneficios es que su cobertura es mayor que la de un radar secundario, y puede ser usado con mayor facilidad en la superficie de un aeropuerto y en espacios aéreos inferiores donde la exactitud de la información es fundamental. Debido a esto, se puede optimizar el uso del espacio aéreo, reducir las restricciones por visibilidad, mejorar la vigilancia en la superficie y aumentar la seguridad. Los equipos de ADS-B pueden ser instalados en los vehículos de superficie y hasta en obstáculos fijos (OACl, 1999).

Cada transmisor ADS-B periódicamente envía en broadcast la posición del vehículo y los otros datos requeridos, los cuales son obtenidos de los sistemas de navegación de abordo. Cualquier usuario, tanto en el aire como en la tierra dentro del rango de cobertura del transmisor ADS-B, puede decidir si recibe y procesa esta información. El transmisor no necesita tener conocimiento de aquellos receptores que están procesando su información. Los requerimientos y características de desempeño de la información ADS-B pueden diferir dependiendo de la clase de espacio aéreo dentro del cual los transmisores deban operar, y el nivel de servicio ofrecido en cada clase de espacio aéreo. El concepto de la tecnología ADS-B es mostrado en la Figura 7.

\section{ADS-B en Colombia}

En 2009, la Aeronáutica Civil de Colombia lanzó el Plan Nacional de Navegación Aérea (PNA), el cual está basado en el Plan Mundial de Navegación Aérea (Aeronáutica Civil,

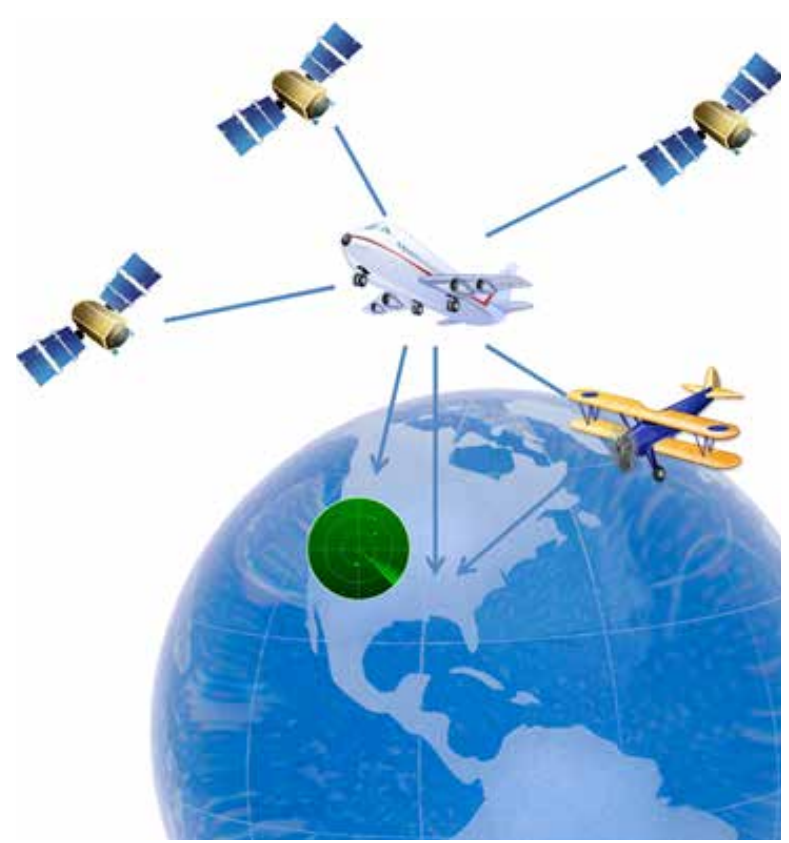

Figura 7. Concepto de ADS-B.

Fuente: elaboración del autor.

2014). Este documento establece los planes para el desarrollo de la aviación en Colombia los cuales deben ser la guía para la comunidad aeronáutica en el corto, mediano y largo plazo.

EI PNA describe en detalle los requerimientos operacionales del espacio aéreo colombiano, con el objetivo de mejorar los niveles de seguridad, los niveles de consistencia, efectividad y eficiencia para el uso del espacio aéreo, y beneficio de la comunidad aeronáutica tanto nacional como internacional. El documento también presenta cuales son los planes de la Autoridad de aviación con respecto a la implementación de las tecnologías apropiadas para la prestación de los servicios de comunicaciones, navegación vigilancia y meteorología aeronáutica, para una adecuada gestión del tránsito aéreo basada en el concepto CNS/ATM (Gómez y Ortíz , 2013).

\section{Estrategia de vigilancia de acuerdo al Plan Nacional de Navegación Aérea (PNA)}

En lugares donde sea necesario y en la medida de lo posible, se deberá brindar el servicio de vigilancia aeronáutica como parte integral del servicio de Control de Tráfico Aéreo a fin de mejorar la seguridad, eficiencia y economía de las operaciones, con las tecnologías disponibles como son: los radares PSR/SSR, el ADS y la Multilateración (MLAT). Se deberá obtener vigilancia total del espacio aéreo Colombiano mediante la combinación del Radar, el ADS y la MLAT, donde el radar y la MLAT serán las herramientas de vigilancia en las áreas de mayor tráfico y para las áreas 
remotas u oceánicas se utilizarán los radares de ruta y el ADS-B. La presentación en las pantallas de visualización integrará datos Radar, ADS y MLAT (Aeronáutica Civil, 2011).

Lo anterior, debido a que los sistemas operativos en la actualidad ya han cumplido su ciclo de vida útil (sensores con más de 15 años y centros de vigilancia ATC con más de 8 años), sus tecnologías no tienen la capacidad de procesar toda la información debido al gran aumento del tráfico aéreo, se hace difícil la consecución de repuestos para la ejecución del mantenimiento y finalmente se han desarrollado actualizaciones en la tecnología con nuevas funcionalidades que hacen más eficiente y amigable la prestación del servicio ATC, las cuales deben ser implementadas a nivel nacional (Aeronáutica Civil, 2011).

ADS-B y MLAT han sido identificadas como la solución para la vigilancia, cubriendo las necesidades de información crítica de los vuelos tanto a pilotos como controladores de tránsito aéreo. A fin de determinar la viabilidad del uso de ADS-B como herramienta de vigilancia en Colombia, es necesario recolectar datos para validar la calidad de los mensajes ADS-B actualmente emitidos, identificar las aeronaves que están equipadas y las zonas de conveniencia para implementar las estaciones terrenas (Aeronáutica Civil, 2011).

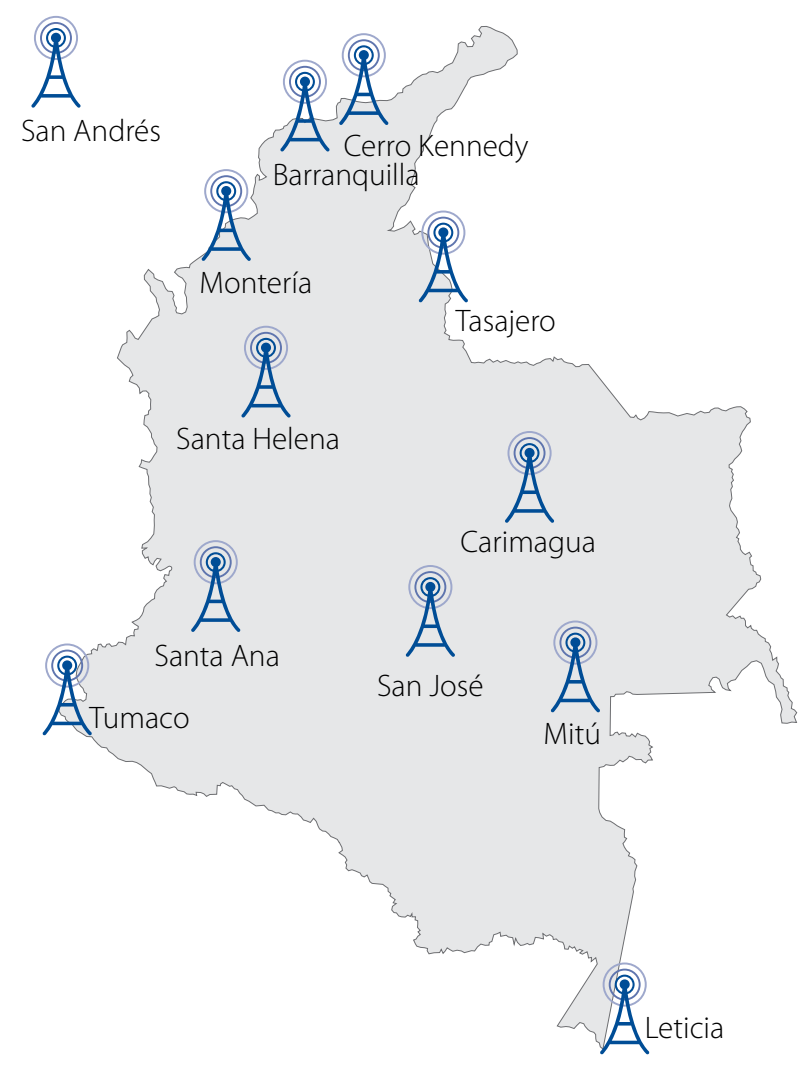

Figura 8. Estaciones terrenas ADS.

Fuente: elaboración del autor.
Durante el año 2015 se ha ido desarrollando la primera fase de la implementación del sistema de vigilancia ADS-B. En esta fase se han implementado 11 estaciones ADS-B en diferentes partes del País, como se puede ver en la Figura 8. Tres de ellas (Cerro Kennedy, Barranquilla, Montería), están integradas al centro de control de Barranquilla, una está integrada a la sala de vigilancia de San Andrés, y las siete restantes (Tumaco, Tasajero, Santa Ana, Mitú, San José del Guaviare, St. Helena y Carimagua) están integradas al centro de control de Bogotá. AdicionaImente, se tiene planeada la instalación de otra estación en la ciudad de Leticia (Amazonas).

El objetivo de esta primera fase del proyecto es proveer el servicio ADS-B a través del territorio colombiano, en niveles superiores en los cuales el tránsito aéreo internacional es predominante. Esto, debido a que las aeronaves que cubren este tipo de rutas, ya cuentan con el equipamiento necesario para establecer un enlace de datos de vigilancia ADS-B. Las estaciones en tierra que están siendo instaladas son suministradas por la compañía INDRA, la cual también ha estado involucrada en proyectos de modernización de los centros de control de tránsito aéreo en Colombia. Una vez se encuentre en funcionamiento el sistema en su totalidad, se espera que funcione como un complemento a los sistemas convencionales de Radar, lo cual dará cobertura a aquellas áreas donde falla la señal Radar. Como la información ADS-B está integrada a los centros de procesamiento de datos Radar, el sistema toma ambas señales, la del Radar y la ADS-B, y presenta un solo dato en la pantalla del controlador de tránsito aéreo, de tal manera que para él, es transparente con que tecnología se está detectando la aeronave.

El protocolo de comunicación usado por las estaciones para recibir la información enviada por las aeronaves, es el "Modo S 1090 Extended squitter". En la actualidad, no existe una regulación definida por la autoridad de aviación civil para el uso de este enlace de datos, pero se espera que para el 01 de Enero de 2020 todas las aeronaves que sobrevuelen el espacio aéreo colombiano tengan instalados los equipos de abordo necesarios para establecer este enlace mediante el cual puedan ser vigiladas por las estaciones terrenas (Aeronáutica Civil, 2014).

\section{Estaciones terrenas ADS-B}

La estación terrena ADS-B está compuesta por una antena, un equipo receptor y una unidad de procesamiento que recibe, procesa y decodifica la información ADS-B y un procesador ADS-B que correlaciona y crea reportes ASTERIX para ser enviados finalmente al sistema ATM. El sistema ADS-B de INDRA tiene tres canales de recepción independientes. En escenarios de cargas pesadas de información, 
esto se traduce en un mejor desempeño a largas distancias (INDRA Company, 2015).

El sistema puede ser configurado desde un simple receptor ADS-B con una única antena omnidireccional con una única unidad de procesamiento, hasta un sistema totalmente redundante incluyendo elementos como antenas sectorizadas y cadenas de procesamiento redundantes. El ADS-B de INDRA está basado en elementos COTS e incluye funcionalidades BITE y CMS que detectan eventos y almacenan los datos relevantes. Como es recomendado en los estándares relativos al ADS-B, las funcionalidades del sistema usan el protocolo SNMP (INDRA Company, 2015).

\section{Conclusiones}

Se estudió la implementación de nuevas tecnologías para la prestación de los servicios a la navegación aérea en Colombia, haciendo énfasis en el sistema de vigilancia ADS-B, en el marco del Plan Nacional de Navegación Aérea para Colombia.

La implementación de nuevas tecnologías para la prestación de los servicios a la navegación aérea a nivel global es una realidad, de la cual Colombia no es ajena. El aumento en las operaciones de tránsito aéreo, hace necesaria la mejora en los procesos y sistemas, por lo que cobra gran importancia el estudio de las condiciones locales para la implementación de tecnologías como ADS-B.

Debido a las condiciones orográficas del territorio colombiano, existen zonas que quedan fuera de la cobertura de la señal de vigilancia radar. Estas zonas pueden ser aprovechadas para la realización de actividades ilícitas sin la vigilancia del Estado colombiano. La implementación de ADS-B, permitirá mejorar la cobertura actual y la vigilancia de estas zonas sin recurrir a la compra e instalación de costosos sistemas de vigilancia Radar.

ADS-B se considera una tecnología de vigilancia colaborativa, debido a que necesita que la aeronave tenga instalado y encendido un transmisor a bordo que informe su posición a las estaciones de tierra. Debido a esto, ADS no puede suplir a un radar primario PSR en su tarea de detectar blancos móviles que no posean o no tengan activado su equipo a bordo, como aeronaves ilícitas u objetos diferentes como un misil de guerra.

Desde la primera edición del plan nacional de navegación aérea, en el año 2009, Colombia ha estado en un proceso de actualización de sistemas para el soporte de los servicios a la navegación aérea tanto para comunicaciones, como para navegación y vigilancia. Con la implementación de las estaciones ADS-B, se da un paso muy importante en la transición desde los sistemas convencionales de vigilancia Radar, a la vigilancia ADS-B, que utiliza sistemas de navegación satelital y transmisión de enlaces de datos entre las aeronaves y las estaciones terrenas.

Actualmente, está en desarrollo la primera fase de todo el proyecto de implementación de ADS-B en Colombia. La Aeronáutica Civil desarrollará el proyecto por fases, debido a que además de la implementación de los equipos en tierra, las aeronaves de cada una de las compañías aéreas deben estar equipadas también con sistemas de vigilancia ADS-B. El Estado colombiano ha determinado un tiempo de cinco años para que todas las aeronaves se equipen con la aviónica necesaria que reporte la posición de la aeronave a través de un enlace de datos "Modo $S$ 1090 Extended Squitter" a las estaciones terrenas ADS-B, de tal forma que la autoridad de aviación civil pueda realizar la vigilancia sobre estas.

Se espera que a mediano plazo, el sistema ADS-B, junto con otras tecnologías de vigilancia como la Multilateración (MLAT), sean un complemento a los radares para propósitos de control de tránsito aéreo.

\section{Agradecimientos}

El autor quiere agradecer a la Aeronáutica Civil de Colombia por su contribución con este artículo. Gracias al ingeniero Pedro Velasco, del grupo de Vigilancia Aeronáutica por su apoyo y aportes en la construcción del mismo.

\section{Referencias}

ADS-B Technologies. (2015). ADS-B Technologies official web site. Obtenido de ¿What is ADS-B? Retrieved from www.ads-b.com

Aeronáutica Civil de Colombia. (2011). Plan de navegacion aérea para Colombia. Servicios e Instalaciones (Vol. 2). Bogotá.

Aeronáutica Civil de Colombia. (2014). Plan de Navegación Aérea para Colombia (Vol. 1). Bogotá.

Aeronáutica Civil de Colombia. (2015). Aeronautical Information Publication AIP. Bogotá.

Aeronáutica Civil de Colombia. (2015). Reglamento Aeronáutico Colombiano RAC. Bogotá.

Airservices Australia. (2012). How ADS-B works.

Farroha, B. (2002). Next Generation Data link Air Traffic Technology International. UK \& International Press.

Gómez , L., \& Ortíz, J. (2012). VHF Data Link communications to provide air traffic services in Colombia. 31st Digital Avionics Systems Conference (DASC). 
CIENCIA Y PODER AÉREO | Revista Científica de la Escuela de Postgrados de la Fuerza Aérea Colombiana | Vol. 10 | Enero - Diciembre de 2015

Gómez, L., \& Ortíz , J. (2013). Modeling and simulation of VDL mode 2 subnet for CPDLC in El Dorado Airport. 32st Digital Avionics Systems Conference, DASC.

Gómez, L., \& Ortíz , J. (2014). Results of a VDL 2 subnet simulation in the approach area to El Dorado Airport. 33st Digital Avionics Systems Conference, DASC.

INDRA Company. (2015). Automatic Dependent Surveillance - Broadcast, ADS-B. Retrieved from http://www.indracompany. com/en/automatic-dependent-surveillance-broadcast-adsb

Hamish, M. (2001). Modern Radar Systems. Artech House, Norwood, MA.

Matamoros, H. (1999). Estudio de CNS para Colombia. Francia: Ecole Nationale de l'a Aviation Civile E.N.A.C

Mahafza, B. (1998). Introduction to radar analysis. ISBN: 0-84931879-3. CRC Press, NY.

Morales López y Juan de Mata. (2001). Sistemas CNS/ATM Vol 2. AENA

OACI. (1999). Manual of air traffic services data link applications, Doc 9694. Montreal.

OACI. (2002). Manual on VHF Digital Link (VDL) Mode 2, Doc 9776. Montreal.

OACI. (2007). Anexo 10: Radares de vigilancia y sistemas anti colisión (Vol. 4). Montreal.

OACI. (2007). Anexo 10: Radio ayudas para la navegacion aérea (Vol. 1). Montreal.

OACI. (2011). Sistemas CNS/ATM de la OACI. [En línea]. Disponible en GREPECAS http://www.lima.icao.int/meetprog /2003/ HRTS/SISTEMAS\%20CNSATM.pdf

OACI. (2012). Página Oficial OACl. Obtenido de CNS-ATM Systems. Retrieved from: www.icao.int/icao/en/ro/rio/execsum.pdf

OACl. (2013). Air Traffic Management Doc 4444. Montreal

Richards, W. R., O'Brien, K. M., \& Dean, C. (2010). New Air Traffic Surveillance Technology. Boeing aero quarterly 2. Retrieved 7 April 2014. 